\title{
Analysis of the Marketing Strategy of Bilibili and the Reasons for Its Success
}

\begin{abstract}
Hansong Tian
The University of Newcastle Australia

Hansong.Tian@uon.edu.au

ABSTRACT

China's video platforms show a good and rapid development momentum in 2019-2021, among which Bilibili is the most rapid. Its number of users has doubled in just two years and it has become one of the most popular video platforms in China. Founded in 2009, Bilibili focuses on ACG subculture. After more than a decade of development and covering more than 7,000 interest circles, Bilibili has gradually become a highly concentrated cultural community and video platform for the young generation in China. This article will use case analysis and comparative analysis to explore the reasons for the success of the Bilibili strategy. The result of the study is that Bilibili has a competitive advantage in the Chinese young generation consumer market by using the product strategy of community mode operation, cheap and simple price strategy and vertical marketing channel strategy. Excellent marketing strategy, high sticky-user groups and community operation mode are the reasons for Bilibili's success.
\end{abstract}

Keywords : ACG subculture, barrage sites, youth, social media

\section{INTRODUCTION}

In 2019-2021, Chinese video platforms have gradually grown and matured. With the continuous development of science and technology and the acceleration of Internet browsing and downloading speed, both traditional video platforms and new integrated video platforms have a certain number of stable and loyal user groups [4]. Especially in 2020, due to the impact of COVID-19, people are increasingly working, studying and entertaining at home, and the number of users of video platforms in China is also on the rise. As the whole industry is booming, the new integrated video platform can attract more users and gain more loyal users compared to the traditional video platform. Although traditional video platforms have actively launched new video content or reduced some member fees in the past two years, there is still a lot of gap between traditional video platforms and new integrated video platforms in terms of user growth and user utilization rate. In the case of Bilibili, Bilibili's user base has doubled from 100 million users in 2019 to 200 million users in 2021, and it has become one of the preferred video platforms for the younger generation of users in China [6]. Many video platforms have grown as a result of the epidemic, gaining more users. However, in terms of its development speed, Bilibili is much faster than other video platforms. Bilibili's huge success in just a few years is inseparable from its marketing strategy and approach. Therefore, this paper will analyze Bilibili's marketing strategy and the reasons for its successful marketing. In the following analysis, it will be divided into two steps. The first step is to analyze the overall marketing strategy adopted by Bilibili for users, and then the author will further analyze the factors of its successful marketing in combination with Bilibili's marketing strategy to explore the reasons for Bilibili's success.

\section{OVERVIEW OF BILIBILI}

Bilibili is a highly concentrated cultural community and video platform for the young generation in China. The website was founded on June 26, 2009. On March 28, 2018, Bilibili was listed on NASDAQ. On March 18, 2020 , Bilibili's total net revenue for the fourth quarter reached RMB 2,007.8 million. On February 25, 2021, Bilibili's total net revenue for the fourth quarter of 2020 reached RMB3.84 billion, an increase of 91\% compared to the same period in 2019 [6]. Bilibili was an ACG (animation, manga, game) content creation and sharing video site in its early days.

At first, the site was only for those users who liked ACG content, providing corresponding content resources 
for these users, and attracting them to do high-quality secondary creation. After more than a decade of development, Bilibili has built an ecosystem of highquality content around users, creators and content. Bilibili has covered more than 7,000 multi-cultural communities of interest. Now Bilibili is not only for users who like ACG content, but for users who like different fields, they can find their interests in Bilibili. Moreover, bilibili has always maintained a good relationship with its users in more than ten years of development. Any user can publish their second creation videos on Bilibili. This has attracted a growing number of users to use bilibili, both as users and as creators of video content. Bilibili has won the first place in the list of "Gen Z Favorite App" and "Gen Z Favorite Panentertainment App" by Quest Mobile Research Institute, and has been selected as one of the top 100 most valuable Chinese brands by "BrandZ" in 2019 [5]. Since Bilibili held its Bilibili Gala: the Most Beautiful Night of 2020 in 2019, Bilibili's development has been moving forward rapidly [5]. Just two years later, the number of users of Bilibili has reached an astonishing 200 million.

\section{BILIBILI'S MARKETING STRATEGY AND FACTORS FOR SUCCESSFUL MARKETING}

\subsection{Bilibili's marketing strategy}

Marketing strategy refers to a series of activities adopted by an enterprise for the selected target market based on customer needs, information on purchasing power and expectations of the business community. These activities are planned and controlled to improve sales performance and the reputation of the manufacturer [1]. The purpose of marketing strategy is to let more customers know a certain brand through product quality, price and promotion, and improve customer satisfaction with the brand, so as to improve product sales of the brand. A good marketing strategy can win a huge competitive advantage from the aspects of products, prices, channels and so on, which brings great benefits to the enterprise and also enhances the image and reputation of the enterprise. Next, this paper will analyze Bilibili's marketing strategy from three aspects: product, price and channel, and understand the advantages Bilibili's marketing strategy has gained in the market.

\subsubsection{Product strategy}

Product strategy is a series of measures and means used by enterprises in the production and sales of products in order to gain advantages in the fierce market competition [1]. For enterprises, the first thing to be clear is what kind of products they can provide to meet the needs of consumers, including specific implementation strategies in trademark, brand, packaging, product positioning, product mix, product life cycle and other aspects. This strategy is also an important part of the marketing mix strategy. The continuous expansion of product extension of modern enterprises is due to the complexity of consumer demand and the white-hot competition. Under the situation of convergence of core functions of products in the current market, which enterprise can meet the interests of consumers more and better, the enterprise can better occupy the market and obtain competitive advantages. Community video platform is the core product of Bilibili at present. Bilibili allows users to participate and build together the sections they are interested in. Bilibili also helps users upload videos they are interested in to share with other users. Users actively participate in discussions and users upload and share videos, which build Bilibili's unique video community model. This model also eliminates the gap between users of traditional video platforms and video uploaders. Users are more likely to leave comments at the bottom of videos, and video uploaders are more able to accept users' feedback and establish a more direct relationship with users. This connection allows video uploaders to create better works that attract more viewers. At the same time, those users who upload videos are also called "up-masters", and up-masters are the main source of Bilibili's content. By the end of 2019, Bilibili had uploaded 3.1 million videos per month, according to data [3]. Bilibili's core products meet users' needs for diversified videos and a harmonious and friendly atmosphere for discussion. In practice, users can find sections in diversified videos that meet their needs, and feel a proper sense of belonging in the atmosphere of community-style discussion. Starting with core products, Bilibili has changed its product strategy of video platform, and made use of the community-type platform model to meet the needs of more users, thus gaining a competitive advantage.

\subsubsection{Price strategy}

Different from other video platforms, Bilibili's charging method is simpler and cheaper. Bilibili integrates its membership system so that users who buy its membership can watch all types of videos without charging extra for individual videos [3]. Bilibili's more straightforward approach makes users more willing to pay for membership than the more complex charging systems of other traditional video platforms. Bilibili sets its prices based on those of its competitors. For example, the same purchase of one-year membership, iQIYI charges 180 yuan, Youku 175 yuan, Bilibili 168 yuan [3]. Bilibili's competitive advantage is reflected through price discounts. At the same time, as Bilibili does not need to buy anything extra once it buys a member, it is also more competitive in terms of charging model compared with other video platforms' complicated charging mechanism. 


\subsubsection{Channel strategy}

Channel strategy is an important part of the whole marketing system, it is of great significance to reduce the cost and improve the competitiveness of enterprises [1]. The choice of marketing channels will directly affect other marketing decisions, such as product pricing. Channel strategy is also an important means for enterprises to successfully explore the market and achieve sales and business objectives. As Bilibili itself is an Internet company, so in terms of channel strategy, Bilibili chooses the vertical marketing channel strategy. The vertical marketing strategy allows users to spend more time on the platform, making it more accurate to find the content they are interested in, thus increasing their durability and immersion. On the contrary, sunken vertical classifications provide a clear and intuitive view of the platform's user data, providing a quick overview of user group portraits. This data is very attractive to advertisers, making it faster and easier for them to invest in ads to improve their platforms' marketing and commercialization capabilities. When the marketing and commercialization capabilities of the platform are enhanced, the product quality and user satisfaction will also increase. Bilibili further strengthens its market competitiveness by choosing vertical marketing channel strategy.

\subsection{Factors of Bilibili's successful marketing}

\subsubsection{Target market of Bilibili}

The demographic segmentation target market of Bilibili is mainly the young user group. As an emerging platform for cultural community communication, the spirit of innovation and youthful image can be reflected in various sections of Bilibili. For example, the homepage of Bilibili usually displays various ACG cultures and trend cultures. Young people are generally more likely to accept and understand Bilibili's corporate philosophy, so Bilibili aims to target young users. [3]. In 2019, Bilibili hosted "Bilibili Evening: the Most Beautiful Night of 2012", a grand gala for contemporary Chinese youth by combining the hottest young topics of the year. Almost all of the participants in bilibili's party are "up masters", and in this way, bilibili further deepens the connection between users. The identity between the user and the video uploader can be changed at any time in Bilibili. Users can create their own video content and become creators who can also interact with other users. This kind of communication and connection can greatly improve the quality of bilibili video content. In the end, the evening made an important contribution to the further development of Bilibili with its high ratings [6]. Bilibili constantly strengthens its target market, so that its users are highly sticky and loyal.

\subsubsection{Market positioning of Bilibili}

Market positioning means that enterprises study users' liking for certain characteristics of products, and build and publicize the unique image of products according to users' preferences. Through this unique image, customers can clearly distinguish between different brands, and they will also choose a unique brand that impresses them. Consequently, the enterprise through the marketing mix to quickly and accurately convey this image to customers, to achieve a certain competitive advantage [2]. In the early stage, Bilibili developed from ACG culture, and in its later development, Bilibili has always taken ACG culture as its publicity image. Over the years, Bilibili has developed outwards and accommodated video content of different cultural types with ACG culture as the centre. Now Bilibili has developed more video categories, but they all retain a certain ACG culture, which makes the number of Bilibili user groups have been steadily increasing. As a whole, Bilibili has a strong characteristic of ACG culture, which makes Bilibili have a very different image from other video websites [6]. When ACG culture is mentioned, users will naturally think of Bilibili. Bilibili impressed many users with its brand image embeded with strong unique style. When users need to watch videos, they will choose Bilibili, a unique video platform. Such a clear market positioning makes the image of Bilibili remembered by more users, in the market competition to gain a greater advantage.

\subsubsection{Analysis of competitors of Bilibili}

For enterprises, it is very important to establish direct competitors and clarify their long-term goals and capabilities. Only by timely judging the changes in the behavior of competitors can enterprises make the most effective responsive measures when threatened by competitors. In China, Bilibili's main competitors are iQIYI, while globally, Bilibili's competitors are YouTube and Niconico.

IQIYI has a very long history of operation and a large user base in China. On June 11, 2019, iQIYI was selected as one of the "2019 Forbes China's Most Innovative Companies". In December 2019, iQIYI was selected as the model 100 brands in the 2019 China Brand Power Ceremony [4]. The market positioning of iQiyi is variety shows, and the target group is about 20-36 years old. By comparing iQiyi and Bilibili, we can find that although iQiyi has a large number of users, the user engagement of IQiyi is low due to the lack of communication atmosphere among users. Bilibili pays more attention to the communication and discussion atmosphere between users, because this enables bilibili's community model to continue to operate. Bilibili is highly innovative and youthful, which increases user engagement and loyalty. Bilibili has high user activity and viscosity, which is also 
more attractive to users compared with IQiyi. Excellent market positioning enables Bilibili to gain a huge advantage in market competition.

YouTube has an even larger user base. On July 17, 2015, YouTube had more than 1 billion users, and nearly a third of all people online in the world watch videos on YouTube every day [4]. YouTube is marketed as a comprehensive, short video genre aimed at almost all target audiences, which means that almost everyone can find videos they are interested in on YouTube. Bilibili's market positioning is similar to bilibili's by comparing oil pipelines with bilibili. But Bilibili has improved users' sense of belonging through a more unique, communitystyle business model. This sense of belonging makes users more loyal, and they are more willing to spend more time on Bilibili, because they can enjoy watching, sharing and discussing videos in their favorite fields. In China, similar users prefer Bilibili to YouTube, which gives Bilibili a competitive advantage.

Niconico, also the source of ACG culture, has more than 48 million registered and more than 2 million paying members as of 2015 [4]. Niconico is also targeting ACG culture, targeting a similar audience to Bilibili. But Niconico is limited by the population of Japan itself and has fewer users overall than Bilibili, giving Bilibili a more competitive advantage by having a larger user base. In general, Bilibili has a great competitive advantage in terms of operation mode, user stickiness and loyalty due to its main target of development in China.

\section{CONCLUSION}

The marketing strategy of Bilibili includes the product strategy through the community mode operation, the cheap and simple price strategy and the vertical marketing channel strategy to occupy the competitive edge in the market. Currently, Bilibili is targeting young people in China. By integrating ACG culture into more new video segments, Bilibili maintains its unique image while attracting more potential young people. Bilibili has become one of the most popular emerging video platforms for young people by operating a unique community-style model. The success factors of Bilibili include: excellent marketing strategy, highly engaged user group, and community-based operation model. These factors combine to make Bilibili successful today.

At present, this paper only makes case analysis and comparative analysis by using second-hand data on the Internet, without further in-depth construction of data analysis model, so there may be some inaccuracy in this paper. In the future, this paper can collect more detailed data and use mathematical models to proofread different video platforms to draw more accurate conclusions. In the future, this paper will focus on the development of video platforms and analyze the reasons for the success of more video platforms.

\section{ACKNOWLEDGMENT}

I would like to express my gratitude to $\mathrm{Ms}$. Su Mengyang and Ms. Zou Zehui for their help and support in this paper. Besides, I would like to give a heart-felt thankfulness to my parents and friends whose support and help make me full of energy to move forward in this research and following studies.

\section{REFERENCES}

[1] Rajagopal. (2019). Contemporary Marketing Strategy: Analyzing Consumer Behavior to Drive Managerial Decision Making. London, England: Springer Nature.

[2] Wedel, M., Kamakura, W. A. (2000). Market Segmentation: Conceptual and Methodological Foundations. Berlin, Germany: Springer.

[3] Yi, C., Shengqi, C., \& Tong, W. (2013). Perspective of Bullet-screen Websites and Bullet-screen clan: a perspective of youth subculture. Youth to explore, (06), 19-24. http://doi: 10.13583 / j. carol carroll nki issn1004-3780.2013.06.006.

[4] Yutong, W. (2020). Homemade play video website and its profit model (a master's degree thesis, Nanjing college of art). Retrieved from https://kns.cnki.net/kcms/detail/detail.aspx?dbcode $=$ CMFD $\&$ dbname $=$ CMFD202101 \& filename $=1020$ 124542.nh\&v=suJKL5vI126rDZt3JyVdoOTB0Wd xOxuYGlpx5VbUPlvFyAGh6XJkqNH1Sf4z9wW $\mathrm{K}$

[5] Yang, T. (2018). (2018). Research on cultural Field Construction of BILIBILI Video website. (eds.) Proceedings of 2018 3rd International Conference on Social Sciences and Social Sciences Humanities (ICSSH2018) (pp.49-61).

[6] Sisi, Y. (2020). Under the perspective of ACG subculture Bilibili user interactive communication studies (master's degree thesis, Jiangxi university of finance and economics). Retrieved from https://kns.cnki.net/KCMS/detail/detail.aspx?filena $\mathrm{me}=1020976878 . \mathrm{nh} \& \mathrm{dbname}=\mathrm{CMFD} 202101 \& \mathrm{db}$ code $=$ cdmd $\&$ uid $=\& \mathrm{v}=$ MDAwNDFyQ1VSN3Vm WU9kdUZ5M2xVN3pLVkYyNUhycS9HTm5Mc DVFYlBJUitmbnM0eVJZYW16MTFQSGJrcVdB MEY= 